\title{
AVALIAÇÃO DA ASSISTÊNCIA MÉDICA PRESTADA NO AMBULATÓRIO DA LIGA DE COMBATE ÀS DST/AIDS - CENTRO DE SAÚDE, S.P., 1994-1997
}

\author{
EVALUATION OF THE CARE PROVIDED BY THE OUTPATIENT CLINIC OF \\ THE LEAGUE OF COMBAT AGAINST STD/AIDS, HEALTH CENTER, S.P., 1994-1997
}

\author{
Deborah M. Ramos ${ }^{1}$; Fábio de P. Turco'; Daniela A. de Moraes ${ }^{1}$; \\ Ricardo C. Cavalli2; Naiara F. Xavier ${ }^{1}$ \& Aldaisa C. Forster ${ }^{3}$
}

\begin{abstract}
${ }^{1}$ Alunos de Graduação da FMRP-USP; ${ }^{2}$ Médico Residente de $2^{\circ}$ ano do HCFMRP-USP; ${ }^{3}$ Docente do Departamento de Medicina Social. Faculdade de Medicina de Ribeirão Preto da Universidade de São Paulo.

CorResPondência: Av. Bandeirantes, 3900 - CEP: 14049-900 - Ribeirão Preto - SP - fax (016) 633-1386 e.mail - acforste@fmrp.usp.br
\end{abstract}

RAMOS DM; TURCO FP; MORAES DA; CAVALLI RC; XAVIER NF \& FORSTER AC. Avaliação da assistência médica prestada no ambulatório da Liga de Combate às DST/AIDS - Centro de Saúde, SP, 1994-1997. Medicina, Ribeirão Preto, 32: 326-334, jul./set. 1999.

RESUMO: Este trabalho analisou a assistência médica prestada pelos alunos da Liga de Combate a DST/AIDS, da Faculdade de Medicina de Ribeirão Preto - USP, no curso de três anos e meio, junto ao Programa de Prevenção e Controle de Aids do Centro de Saúde Escola. Foi utilizada a metodologia de avaliação em saúde (processo e resultados), que contemplou o estudo da assistência médica em um período contínuo de seguimento, sendo analisados os diagnósticos formulados, os exames laboratoriais e complementares prescritos, as medidas curativas e de promoção da saúde e prevenção de doenças. Foram estudados sessenta e seis (66) indivíduos, sendo $48,5 \%$ comunicantes de portadores do Vírus da Imunodeficiência Humana ou de doentes de aids, $27,3 \%$ portadores do HIV e $24,2 \%$ de doentes de aids. Houve maioria de pacientes masculinos, embora a relação homem:mulher cresça de 1:1, 2:1 e 3,5:1 no sentido comunicantes, portadores e doentes, com uma frequência modal de 40,9\% na faixa etária de trinta (30) e trinta e nove (39) anos, o grau de escolaridade e as ocupações foram característicos de nível socioeconômico baixo. Formularam-se diagnósticos de deficiência da imunidade celular, exposição a doenças transmissíveis (aids, tuberculose e outras doenças infecciosas), infecções respiratórias agudas, doenças sexualmente transmissíveis, parasitoses, entre as principais. Em média, a clientela permaneceu 3,2 meses em seguimento, recebeu 2,4 consultas e 47,7\% dela abandonou 0 atendimento. Os alunos participaram ativamente da pesquisa; a metodologia e as informações quantitativas contribuíram para a avaliação da qualidade da assistência médica, tendo o serviço de saúde correspondido como alternativa institucional para formação e assistência mais próxima da comunidade.

UNITERMOS: Avaliação de Processos e Resultados (Cuidados de Saúde). Assistência Médica. Educação Médica.

\section{INTRODUÇÃO}

O reconhecimento da Síndrome da Imunodeficiência Adquirida ocorreu por volta do início da década de oitenta (80), nos Estados Unidos da América, quando houve aumento do número de pacientes adultos, do sexo masculino, homossexuais e moradores nas cidades de São Francisco e Nova York, que apresentavam queda da eficiência do sistema imune, sarcoma de Kaposi e pneumonia por Pneumocystis Carinii ${ }^{(1)}$. 
Em 1983, houve a identificação do agente etiológico como um retrovírus, com material genético RNA, denominado, universalmente, como HIV - Vírus da Imunodeficiência Humana, apresentando-se em dois subtipos diferentes, HIV-1 e HIV-2.

Ainda em relação às especificidades do HIV, tem sido demonstrada a grande heterogeneidade de suas características biológicas, sorológicas e moleculares. O subtipo HIV-2 tem menor virulência e o subtipo 1 apresenta seis variantes (subtipos de HIV-1 A-F) e, a principio, uma distribuição diferente dos subtipos, nos países e continentes ${ }^{(1)}$.

A transmissão do HIV pode ocorrer durante as relações sexuais (vaginais, anais e orais), nos contatos com sangue e hemoderivados (tratamentos, drogas endovenosas e ferimentos perfurocortantes, nos acidentes ocupacionais, em profissionais de saúde e durante o período perinatal (vertical), no final da gestação (transplacentária), durante o trabalho de parto e amamentação $^{(1)}$. Embora o vírus tenha sido encontrado na maioria dos líquidos e secreções corporais, a forma livre é muito frágil, sendo a forma intracelular mais importante para a sua transmissão.

Nesse aspecto, a contribuição da Epidemiologia foi marcante, pois anterior à descoberta do vírus, foi possível conhecer os grupos da população mais atingidos, as diversas maneiras de transmissão e as medidas de promoção e prevenção que efetivamente podem controlar a doença na população.

Os fatores que aumentam o risco de transmissão do HIV são: alta viremia; imunodeficiência avançada; relação sexual anal receptiva; relação sexual durante a menstruação; concomitância de doenças sexualmente transmissíveis (DST), principalmente as que causam úlceras genitais (cancróide, sífilis, herpes simples) ${ }^{(2)}$.

A Organização Mundial de Saúde (OMS) estimou, "para o ano 2000, um número acumulado de infectados pelo HIV (adultos e crianças) ao redor de trinta (30) a quarenta (40) milhões"(3). Segundo o Programa Global de $\operatorname{AIDS}^{(2)}$, até junho de 1997, estimaram-se cento e trinta e três mil (133.000) casos para cento e dez mil, oitocentas e setenta e duas (110.872) notificações de aids para o Brasil, que passou a ocupar posição entre os quatro países com maior número de casos registrados. Esta posição desloca-se para quadragésimo e quinquagésimo $\left(40^{\circ}\right.$ e $\left.50^{\circ}\right)$ postos, quando se faz referência ao coeficiente de incidência ou a taxas populacionais.

A aids foi identificada, pela primeira vez, no país, em 1982, quando sete pessoas de prática homo/ bissexual foram diagnosticadas com a doença. Um caso foi reconhecido, retrospectivamente, no Estado de São Paulo, como ocorrência de 1980, concluindose que a introdução do vírus ocorreu por volta da década de setenta $(70)^{(2)}$.

A epidemia de aids distribui-se de forma heterogênea entre as regiões do país, concentrando-se a maioria dos casos na Região Sudeste - 69\% dos casos notificados entre 1994 e 98. O Estado de São Paulo apresentou, em quatro períodos analisados - 1980 a 1986; 1987 a 1989; 1990 a 1992 e 1993 a 1997 - a maior incidência média (x 100000 habitantes) do país, taxas de 0,$6 ; 8,0 ; 20,0$; e 25,0, respectivamente $^{(4)}$. No início da epidemia, os municípios mais populosos (mais de um (01) milhão de habitantes) eram responsáveis por $78 \%$ das notificações de aids (52\% em 1992), sendo que dois mil, oitocentos e catorze (2814) municípios "notificaram pelo menos um (01) caso à Coordenação Nacional de Doenças Transmissíveis e AIDS até 30 maio de $1998^{\prime(4)}$. O aumento dos casos entre os heterossexuais, além de interiorizar a epidemia no país, provocou também uma maior exposição das mulheres à doença, o que refletiu, na distribuição dos doentes em relação ao sexo (masculino/feminino); a razão de sexo diminuiu de 23:1 para 2:1, respectivamente, nos anos de 1984 e $1997^{(2)}$.

Em Ribeirão Preto, na década de oitenta (80), havia registrados 17,7 casos, em média, número que cresceu para 158,7, em 1990, atingindo 258,3 entre 1993 e $1995^{(2)}$.

Ortúzar Menesia ${ }^{(5)}$, ao discutir aspectos epidemiológicos da aids em Ribeirão Preto, considerou: o elevado coeficiente de incidência acumulada (550, 2/100 mil, em 1998) da doença no município, colocouo em segunda ( $\left.2^{\mathrm{a}}\right)$ posição no Estado de São Paulo e em quarta (4a) no Brasil; o padrão diferenciado da relação homem: mulher, entre os casos, com valores próximos a quatro (04) até recentemente, diminuiu para 2,6; o grupo etário mais afetado compreendeu as pessoas entre vinte (20) e trinta e quatro (34) anos, com tendência a crescer no grupo acima de trinta (30) anos; o predomínio da categoria de transmissão foi o de uso "de drogas ilícitas endovenosas, durante todo o período de estudo".

O diagnóstico sorológico consiste na detecção dos anticorpos anti - HIV, desenvolvidos nas duas primeiras semanas, ou até os três meses após a exposição ao vírus ${ }^{(4)}$. 
Os testes mais utilizados são os da detecção de anticorpos pela técnica de ensaio imunoenzimático (EIA), denominados de ELISA. Sob o enfoque epidemiológico, é fundamental conhecer o valor preditivo dos testes diagnósticos para a identificação dos verdadeiros positivos e negativos, que, no caso da aids, assume grande importância devido ao impacto social da doença. Estudo realizado por Ruffino-Netto et al. ${ }^{(6)}$ comparando três "kits" comerciais para pesquisa de anticorpo anti-HIV-1 quanto à co-positividade, conegatividade e precisão, obteve valores de sensibilidade de $84 \%$, especificidade de $93 \%$ e a maior taxa de concordância entre dois testes $(93 \%)$.

As manifestações clínicas após a infecção pelo HIV são muito variadas. Nesse sentido, foram elaboradas classificações para contemplar as características clínicas, a duração aproximada e os achados laboratoriais.

O Centro para o Controle de Doenças dos Estados Unidos (CDC) combinou os achados das manifestações clínicas e laboratoriais, disponíveis na época, para elaborar o mapeamento epidemiológico da aids e a classificação de 1986. Em 1993, surgiu nova classificação que incluiu o estado imunológico do paciente, por meio da contagem de linfócitos CD4, possibilitando a avaliação clínica e epidemiológica. Esta última refere-se a pacientes adultos, prevendo-se outra classificação para as crianças devido a dificuldade de definir a doença nesse segmento ${ }^{(4)}$.

Como parte do tratamento, temos duas classes de drogas liberadas, que são as inibidoras da transcriptase reversa e as inibidoras da protease, o que foi estipulado de acordo com a contagem de linfócitos, em especial CD4+e outros dados laboratoriais ${ }^{(4)}$.

É possível perceber serem a infecção HIV e a doença aids motivos de grande preocupação para os profissionais de saúde e a sociedade como um todo. A síndrome tem mostrado ser campo profícuo para o desenvolvimento dos aspectos técnicos e amplos da assistência aos pacientes, da capacitação para outras habilidades de promoção e prevenção, e para permitir a familiarização com o trabalho em equipe e oportunidades de comunicação com pacientes, contatos/familiares de pacientes e comunidade em geral.

Assim, os alunos de Medicina escolheram a síndrome HIV/AIDS como um campo para o aprendizado que ultrapassa os limites das atividades da educação formal e dos serviços de saúde, especialmente do hospital universitário. Fundaram a Liga de Combate a DST/AIDS, em 1993, à semelhança de outros grupos de alunos que, sob orientação docente da Faculdade de Medicina de Ribeirão Preto da Universidade de São Paulo, vêm buscando, em atividades extracurriculares, oportunidades para ampliar a sua formação, utilizando outros espaços de educação favoráveis a mudanças de atitudes. Nas atividades das ligas, floresce um tipo de trabalho em saúde, que fortalece vínculos entre os alunos/supervisores/docentes e a comunidade.

A Liga DST/AIDS tem objetivos de assistência, pesquisa e ensino e vem proporcionando aos alunos a oportunidade de participar de atividades de extensão em serviços de saúde, escolas, casas de apoio a pacientes e de grupos comunitários.

Este trabalho surgiu do interesse que os alunos, vinculados à diretoria da Liga DST/AIDS, tinham em avaliar as atividades do ambulatório da Liga do Centro de Saúde Escola (CSE) da Faculdade de Medicina de Ribeirão Preto - USP (FMRP - USP) quanto aos objetivos preconizados pelo regimento da mesma, e complementar sua formação na área de avaliação em saúde. Desejavam conhecer a qualidade da atenção à saúde, promovida no âmbito da Liga e fomentar, entre os integrantes mais novos, a realização de outros estudos comparativos.

Pretendeu-se, assim, analisar a assistência médica prestada pelos alunos da Liga de Combate às DST/AIDS junto ao Programa de Prevenção e Controle de Aids do CSE da FMRPUSP.

\section{MATERIAL E MÉTODOS}

O Programa de Controle e Prevenção de Aids do CSE procura desenvolver uma abordagem médica e de saúde, com ênfase nas atividades de promoção da saúde e prevenção de doenças aos comunicantes de portadores do HIV, aos portadores do HIV e aos doentes de aids, cujo estado de saúde permite seguimento ambulatorial.

O programa possui uma equipe multiprofissional e atua em uma área distrital no Município de Ribeirão Preto denominada de Distrito Saúde Escola Sumarezinho. O CSE participa do Sistema Municipal de Saúde de Ribeirão Preto e está estruturado segundo níveis de assistência, resolubilidade diagnóstica e terapêutica e mecanismos de referência e contra-referência ${ }^{(7)}$, além de outras características descritas por Oliveira et al. ${ }^{(8)}$. O Ministério de Saúde definiu, recentemente, diretrizes de política assistencial à aids, estimulando a estruturação de uma rede de serviço de 
assistência especializada (SAE), da qual o CSE "Dr Joel D. Machado" vem fazendo parte desde 1994. Esses serviços devem ser constituídos por equipes multidisciplinares e "oferecer assistência humanizada e de qualidade, baseada na atenção integral do paciente e de sua família" (7). A Liga de DST/AIDS seleciona a sua clientela dentre a demanda desse serviço de saúde.

O CSE conta ainda com um serviço de Vigilância Epidemiológica, que notifica e investiga os casos detectados pelas Unidades Básicas de Saúde (nove (09) UBSs) existentes em sua área de abrangência distrital.

Utilizou-se a metodologia de avaliação de programas de saúde pública em serviços ambulatoriais, procurando-se apreender o processo de assistência médica como parte das ações programáticas em saúde.

Propôs-se o seguinte "desenho" de avaliação em saúde:

- observar o seguimento dos pacientes em um período contínuo dentro do programa;

- estudar a acessibilidade da demanda às ações programáticas;

- a qualidade da assistência médica prestada.

Realizou-se a análise retrospectiva dos prontuários dos pacientes atendidos, ou em seguimento, no ambulatório da Liga de DST/AIDS, no período de 1994 a julho de 1997, dos quais se obtiveram as seguintes informações:

1. do paciente - idade, sexo, ocupação e escolaridade;

2. do atendimento médico - data da primeira $\left(1^{\mathrm{a}}\right)$ consulta; pesquisa de anticorpos para HIV; data do primeiro $\left(1^{\circ}\right)$ resultado positivo da pesquisa de anticorpos contra HIV a partir do seguimento na Liga; classificação do paciente em relação à síndrome (classificação de 1986); motivo da procura do programa; queixa; todos os diagnósticos formulados e/ou confirmados clinicamente durante o seguimento; tempo de seguimento no programa; número de consultas realizadas; e condição de saída.

Foram também coletadas informações sobre os exames laboratoriais e complementares, realizados segundo normas do ambulatório da Liga. Foi feita análise cuidadosa dos diagnósticos formulados no prontuário de cada paciente, dos exames laboratoriais e complementares utilizados segundo os critérios do ambulatório da liga, do tratamento e das medidas profiláticas recomendadas.

O ambulatório da Liga padronizou, para o iní- cio de seguimento, os exames laboratoriais e complementares e, caso o paciente necessite de avaliação de nível terciário, é encaminhado à Unidade Especial de Tratamento de Doenças Infecciosas (UETDI) do HCFMRPUSP.

A coleta, a codificação dos dados e dos diagnósticos foram feitas segundo a orientação dos instrumentos de pesquisa (formulário e manual de preenchimento) e das normas da Classificação Internacional das Doenças, $9^{a}$ revisão, 1975.

O processamento eletrônico dos dados foi realizado no Centro de Processamento de Dados Hospitalares do Departamento de Medicina Social da FMRPUSP.

\section{RESULTADOS E DISCUSSÃO}

Um total de sessenta e seis (66) pacientes ingressou no ambulatório do CSE e foi seguido pela Liga no período de 1994 a julho de 1997, e é o objeto deste estudo. Esses indivíduos foram classificados na primeira $\left(1^{\mathrm{a}}\right)$ consulta em: comunicantes de portadores do HIV e de doentes com aids - trinta e dois (32) casos; portadores do HIV - dezesseis (16) casos e doentes com aids - dezoito (18) casos.

A maioria $(56,0 \%)$ da população estudada estava na faixa maior de trinta (30) anos, encontrando-se $16,7 \%$ de adolescentes $6,1 \%$ com menos de quinze (15) anos e $10,6 \%$ na faixa de quinze (15) a dezenove (19) anos) e nenhum paciente com mais de cinqüenta e nove (59) anos (Tabela I). Quanto ao sexo, 63,6\% eram pacientes masculinos, em relação numérica superior em todas as faixas etárias, exceto entre os menores de quinze (15) anos e entre aqueles com cinqüenta (50) a cinqüenta e nove (59) anos. O predomínio do sexo masculino (62\%) na maioria das categorias diagnósticadas, inclusive na de aids, foi encontrado em estudo ${ }^{(9)}$ de demanda (ambulatório e internação) de um Departamento de Doenças Infecciosas no Estado do Rio de Janeiro, no período de 1965 a 1994.

O grau de escolaridade da clientela foi baixo, $68,2 \%$ tinham o primeiro grau incompleto, havendo apenas cinco (05) pacientes que cursaram os $2^{\circ}$ e $3^{\circ}$ graus (Gráfico 1). A clientela que procura os serviços públicos, em geral, tem baixa escolaridade, como observaram César \& Tanaka $^{(10)}$ em inquérito populacional na Região Metropolitana de São Paulo.

No tocante à ocupação, 21,2\% eram aposentados e inativos (aposentados por motivo da doença); 
9,1\% desempregados; $7,6 \%$ estudantes; $7,6 \%$ donas de casa; $7,6 \%$ trabalhadores de hotéis e os demais $(46,9 \%)$ referiram outras ocupações, neste caso, a maioria no setor de prestação de serviços.

Percebe-se, assim, constituirem, os pacientes atendidos pela Liga, no período estudado, um segmento com nível socio-econômico baixo.

A análise da escolaridade da demanda, segundo os três grupos, mostrou a mesma tendência do total, ou seja, referiram $1^{\circ}$ grau incompleto e completo vinte e sete $(27)(81,2 \%)$ pessoas entre os comunicantes, 93,8\% dos portadores do HIV e 94,4\% dos doentes (Gráfico 1).
Os comunicantes distribuem-se de forma semelhante em homens e mulheres, no total e em todas as faixas etárias, exceto entre os menores de quinze (15) anos e na faixa de vinte a vinte e nove (20-29) anos de idade. Entre os portadores do HIV, o número de homens (11) é o dobro do de mulheres (5) no total e nas faixas etárias de vinte a vinte e nove (20-29) e de trinta a trinta e nove (30-39) anos, enquanto entre os doentes de aids (catorze (14) homens e quatro (04) mulheres) os casos concentraram-se nas faixas etárias de trinta a trinta e nove (30-39) anos (onze (11) pessoas) e de vinte a vinte e nove (20-29) anos (cinco (05) pessoas), havendo sempre maioria masculina (Tabela I).

\begin{tabular}{|c|c|c|c|c|c|c|c|c|}
\hline \multirow{3}{*}{$\begin{array}{c}\text { Faixa Etária } \\
<15\end{array}$} & \multicolumn{2}{|c|}{ Comunicante } & \multicolumn{2}{|c|}{ Portador de HIV } & \multicolumn{2}{|c|}{ AIDS } & \multirow{2}{*}{\multicolumn{2}{|c|}{ Total }} \\
\hline & $M$ & $\mathrm{~F}$ & $M$ & $\mathrm{~F}$ & M & $\mathrm{F}$ & & \\
\hline & 1 & 3 & 0 & 0 & 0 & 0 & 4 & $(6,1 \%)$ \\
\hline $15-19$ & 3 & 2 & 1 & 1 & 0 & 0 & 7 & $(10,6 \%)$ \\
\hline $20-29$ & 5 & 2 & 4 & 2 & 4 & 1 & 18 & $(27,3 \%)$ \\
\hline $30-39$ & 5 & 5 & 4 & 2 & 8 & 3 & 27 & $(40,9 \%)$ \\
\hline $40-49$ & 3 & 2 & 2 & 0 & 2 & 0 & 9 & $(13,6 \%)$ \\
\hline $50-59$ & 0 & 1 & 0 & 0 & 0 & 0 & 1 & $(1,5 \%)$ \\
\hline Total & 17 & 15 & 11 & 5 & 14 & 4 & 66 & $(100,0 \%)$ \\
\hline
\end{tabular}

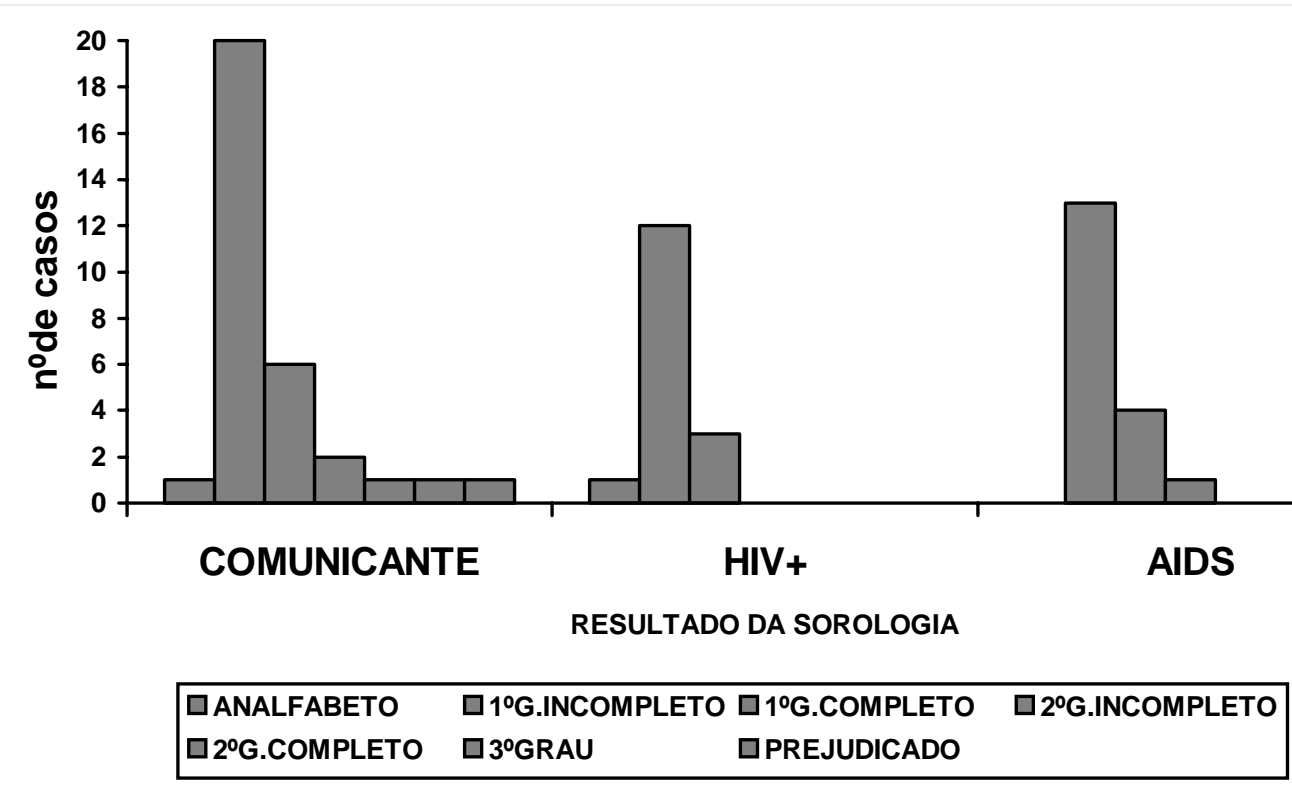

Gráfico 1 - Distribuição dos pacientes segundo o grau de escolaridade e o grupo, Liga de DST/AIDS, CSEFMRPUSP, Ribeirão Preto, 1994-1997. 
A relação de masculinidade evoluiu de 1:1 entre os comunicantes, para 2:1 entre os portadores de vírus e 3,5:1 entre os doentes. Em Ribeirão Preto, a tendência da relação homem: mulher, nos casos de aids, é diferente da encontrada em outros locais do país. No início da epidemia, isso era explicado devido ao consumo de drogas endovenosas por pessoas de ambos os sexos, e, nos últimos anos, provavelmente, também ao aumento da transmissão heterossexual ${ }^{(5)}$.

É possível perceber a mudança do perfil epidemiológico da aids e a necessidade de contar com serviços que disponham também de atendimento ginecológico e pediátrico. Nesse aspecto, a existência de equipe multidisciplinar/multiprofissional, vinculada ao programa e à Liga - médico infectologista, enfermeiro, assistente social, psicólogo, psiquiatra, dentista, etc., vem possibilitando atenção de conotação mais integral, descentralização das ações e acessibilidade aos serviços de saúde, representando condições favoráveis à formação e capacitação de recursos humanos para assistência alternativa aos pacientes com aids ${ }^{(7)}$.

Foram formulados duzentos e noventa e dois (292) diagnósticos para um total de sessenta e seis (66) pacientes, em média 4,4 diagnósticos por paciente, crescendo a relação no sentido dos grupos comunicantes $(2,2)$, portadores do HIV $(6,1)$ e doentes $(6,9)$.

Entre os pacientes comunicantes, os diagnósticos registrados eram compatíveis com situação de risco à doença: contatos com pessoas com aids, exposição familiar a doenças infecciosas, e doenças sexualmente transmissíveis (DST). Gir et al. ${ }^{(11)}$ encontraram que $65,9 \%$ dos pacientes expostos ao HIV, pertencentes às outras categorias que não sexual, apresentavam uma ou mais DST. Registraram-se, também, diagnósticos mais comumente encontrados na população geral, que procura serviços de saúde, como: diabetes, exame médico geral, anemia, além de hipertensão arterial, infecções respiratórias, etc, incluídos aqui em "outros" na Tabela II. Constatou-se, no ambulatório, preocupação em detectar situações de risco à saúde da demanda e diagnosticar as DST, em especial aquelas com lesões ulcerosas nos genitais, que favorecem a transmissão do HIV. Dessa forma, os serviços aumentam a "capacidade de assistir à maior demanda" e facilitar o acesso dos pacientes com suspeita de enfermidades sexualmente transmissíveis" $(11,12)$.

Os pacientes portadores do HIV e doentes apresentaram diagnósticos de deficiência da imunidade celular, havendo, entre os doentes, diagnósticos que abrangeram maior número de aparelhos e órgãos comprometidos (Tabelas III e IV). O grupo "outros" diag- nósticos, entre os portadores do HIV, foi composto por problemas de saúde ou sintomas/sinais em baixas frequiências, apontando para um menor comprometimento da saúde (cefaléia tensional, tosse, dor articular, taquicardia, diarréia infecciosa, etc). Os doentes apresentaram menor número de diagnósticos em "outros", porém podendo representar riscos para a saúde dos pacientes (convulsões, cefaléia, hepatite viral, neoplasma de pele, escabiose, meningite, etc).

\begin{tabular}{|lcc|}
\hline \multicolumn{1}{|c|}{$\begin{array}{l}\text { Tabela II - Diagnósticos em pacientes comunican- } \\
\text { tes, Liga DST/AIDS, CSE - FMRPUSP, }\end{array}$} & 1994 & - 1997 \\
\hline \multicolumn{1}{|c|}{ Diagnósticos } & № & $\%$ \\
\hline Contatos com pessoas com AIDS & 18 & 25,7 \\
\hline Doenças sexualmente transmissíveis & 5 & 7,1 \\
\hline Exame médico geral & 5 & 7,1 \\
\hline Diabetes & 4 & 5,7 \\
\hline $\begin{array}{l}\text { Exposição familiar a doenças } \\
\text { infecciosas }\end{array}$ & 3 & 4,3 \\
\hline Anemia não especificada & 3 & 4,3 \\
\hline Outros & 32 & 45,7 \\
\hline Total & 70 & 99,9 \\
\hline
\end{tabular}

Tabela III - Diagnósticos em portadores do HIV, Liga DST/AIDS, CSE-FMRPUSP, 1994 - 1997

\begin{tabular}{|lrr|}
\hline \multicolumn{1}{|c}{ Diagnósticos } & \multicolumn{1}{c}{${ }^{\circ}$} & \multicolumn{1}{c|}{$\%$} \\
\hline Deficiência da imunidade celular & 20 & 20,4 \\
\hline $\begin{array}{l}\text { Afecções inflamatórias e outras da } \\
\text { pele }\end{array}$ & 9 & 9,2 \\
\hline Infecções respiratórias agudas & 8 & 8,2 \\
\hline Afecções do aparelho digestivo & 4 & 4,1 \\
\hline Amenorréia e transtorno menstrual & 4 & 4,1 \\
\hline Parasitose intestinal & 4 & 4,1 \\
\hline $\begin{array}{l}\text { Contato com pacientes com aids ou } \\
\text { tuberculose }\end{array}$ & 3 & 3,0 \\
\hline Infecções de dentes e gengiva & 3 & 3,0 \\
\hline Escabiose & 3 & 3,0 \\
\hline Diabetes & 3 & 3,0 \\
\hline Leucorréia & 3 & 3,0 \\
\hline Outros & 34 & 34,7 \\
\hline Total & 98 & 100,0 \\
\hline
\end{tabular}


Tabela IV - Diagnósticos em pacientes com aids, Liga DST/AIDS, CSEFMRPUSP, 1994 - 1997

\begin{tabular}{|lrr|}
\hline \multicolumn{1}{|c}{ Diagnósticos } & № & \% \\
\hline Deficiência da imunidade celular & 22 & 17,7 \\
\hline Monilíase oral & 7 & 5,6 \\
\hline Parasitoses & 7 & 5.6 \\
\hline Infecções respiratórias agudas & 7 & 5,6 \\
\hline Afecções inflamatórias e outras da & 6 & 4,8 \\
\hline
\end{tabular}

pele

\begin{tabular}{lll}
\hline Doenças Sexualmente Transmissíveis & 6 & 4,8 \\
\hline
\end{tabular}

Tuberculose pulmonar e das menin- 64,8 ges

\begin{tabular}{|lcc|}
\hline Pneumonia & 5 & 4,0 \\
\hline Tinha das unhas & 4 & 3,2 \\
\hline Diarréias & 4 & 3,2 \\
\hline Outras doenças da traquéia e brôn- & 4 & 3,2 \\
\hline
\end{tabular}
quios

\begin{tabular}{|lrr|}
\hline Herpes & 4 & 3,2 \\
\hline Afecções do trato urinário & 4 & 3,2 \\
\hline Fissura e fístula anal & 3 & 2,4 \\
\hline Visão subnormal em ambos olhos & 3 & 2,4 \\
\hline $\begin{array}{l}\text { Leucoplasia da mucosa bucal e } \\
\text { doenças dos tecidos moles da boca }\end{array}$ & 3 & 2,4 \\
\hline $\begin{array}{l}\text { Contato com pessoas com aids ou } \\
\text { tuberculose }\end{array}$ & 3 & 2,4 \\
\hline $\begin{array}{l}\text { Outros } \\
\text { Total }\end{array}$ & 26 & 21,0 \\
\hline
\end{tabular}

Relacionando, para cada ano do período de estudo e grupo, o número de consultas e o número de pacientes em seguimento, obtivemos, para os comunicantes, o número médio de 2,4 consultas e 3,2 meses de seguimento; para os portadores de HIV 3,7 consultas e 4,5 meses; e, para os doentes 3,7 consultas e 4,6 meses de seguimento. Em resumo, seguimento ambulatorial de curta duração, provavelmente, se deve à busca de diagnóstico do HIV por grupos marginais. Pode também ser considerado uma opção de acesso para assistência para os portadores do HIV e comunicantes dos portadores e dos doentes em busca de orientação/medidas de promoção da saúde e prevenção de doenças. No Brasil, o Ministério da Saúde propõe um programa alternativo de assistência à aids $^{(7)}$, em consoante às recomendações, em outros países, de "reorientar os serviços de saúde para ampliar a acessibilidade e sensibilidade" e prover cuidados adequados "às necessidades atuais de saúde da população"(12).

Quanto à condição de saída, apenas entre os comunicantes houve alta do seguimento, observando-se $47,7 \%$ de abandono nos três (03) grupos e dois (02) óbitos entre os doentes, cinco (05) pacientes encaminhados a serviços de maior nível hierárquico de assistência e outros cinco (05) transferidos. Cerca de $19,7 \%$ dos pacientes mantiveram seguimento no ambulatório até o final do período de estudo (Gráfico 2).

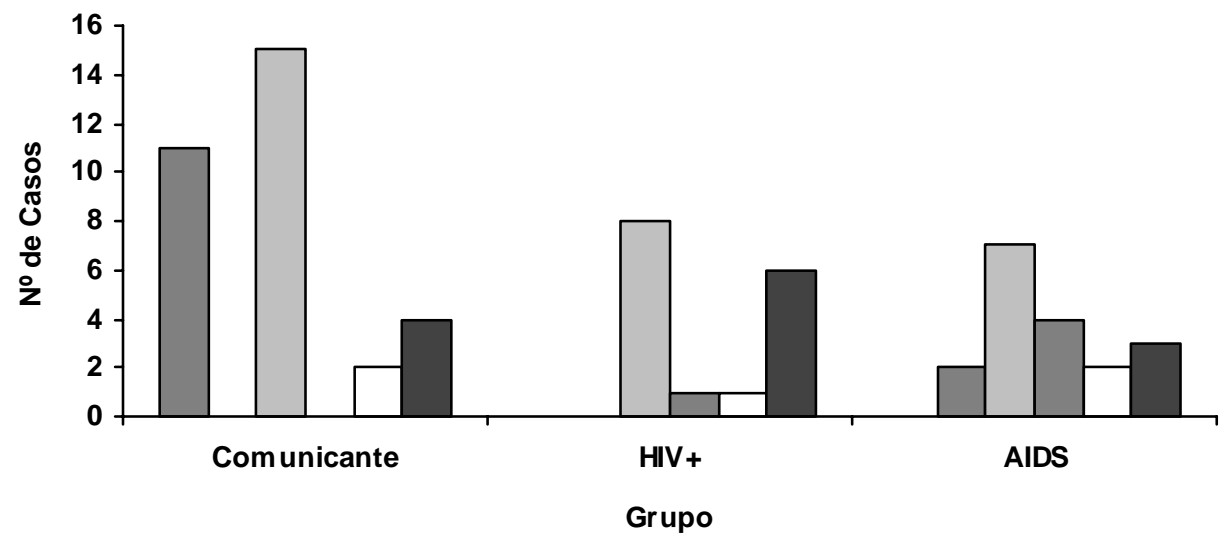

$\square$ Alta $\square$ Óbito $\square$ Abandono $\square$ Referência $\square$ Transferência $\square$ Em seguimento

Gráfico 2 - Distribuição de pacientes segundo a condição de saída e o grupo, Liga DST/AIDS, CSEFMRPUSP, Ribeirão Preto, 1994-1997. 


\section{CONCLUSÕES}

A metodologia de avaliação em saúde proposta, organizada em três itens, abordando o processo do trabalho médico em um período contínuo; a acessibilidade dos pacientes e a condição de saída; e a qualidade da assistência permitiram o cumprimento do objetivo do estudo.

No tocante à qualidade da assistência, verificaram-se diagnósticos clínicos compatíveis com os níveis primário e secundário, o acesso aos recursos laboratoriais, quando necessários, e a investigação laboratorial para outras doenças sexualmente transmissíveis. Observou-se, também, maior integração na assistência devido aos contatos dos alunos e profissionais dos serviços CSE e UETDI - HC-FMRP-USP, possibilitando que os pacientes tivessem acesso aos serviços de apoio ao diagnóstico e terapêutica, sem interromper o seguimento no CSE (serviço mais próximo da comunidade).

O número médio de diagnósticos registrados cresceu com a gravidade do estado da doença e observou-se a preocupação em valorizar a clínica e os recursos laboratoriais na formulação dos diagnósticos e seguimento da clientela.

O tempo de seguimento e a média do número de consultas foram relativamente baixos, provavelmente devido ao fato de ser o grupo de comunicantes mais numeroso que os demais e constituir uma demanda específica à realização do teste ELISA.

As características da clientela - comunicante, portadores do HIV e doentes em estado de saúde mais conservado - e o contato com profissionais de saúde menos especializados contribuem para a formação médica mais adequada ao desenvolvimento das propostas de promoção da saúde e prevenção da aids, recomendadas pelas autoridades de saúde pública ${ }^{(12)}$.

\section{AGRADECIMENTOS}

Os autores agradecem a contribuição inicial do Dr. Jayter Silva de Paula, componente da diretoria da Liga DST/AIDS, em 1996, à secretária, Celia R. Lattaro Marino, aos técnicos de informática e codificação, Rosane Monteiro, Maria Goulart V. Lemos de Almeida, Gilmar Mazer e Dulce Helena de Brito, pela colaboração na elaboração dos instrumentos de pesquisa e processamento eletrônico dos dados da pesquisa.

RAMOS DM; TURCO FP; MORAES DA; CAVALLI RC; XAVIER NF \& FORSTER AC. Evaluation of the care provided by the outpatient clinic of the League of Combat Against STD/AIDS, Health Center, S.P., 19941997. Medicina, Ribeirão Preto, 32: 326-334, july/sept. 1999.

ABSTRACT: The medical care provided by the students of the League of Combat against STD/AIDS belonging to the Faculty of Medicine of Ribeirão Preto - USP over a period of three and a half years was evaluated. We used the method of health evaluation based on the study of medical care during a continuous period of monitoring. The aim was to assess the quality of the care provided, analyzed in terms of the diagnoses formulated, of laboratory and complementary tests, of curative measures and of health promotion and disease prevention. A total of 66 individuals were studied, $48.5 \%$ of them being communicants of patients with HIV and AIDS, $27.3 \%$ HIVinfected patients, and $24.2 \%$ patients with AIDS. Most patients were males, although the man:woman ratio has been growing from 1:1, 2:1 and 3.5:1 in the following order: communicants, infected subjects and patients, with a modal frequency of $40.9 \%$ in the 30 to 39 year age range. Educational level and occupation were characteristic of a low socioeconomic level. The following diagnoses were made: cell immune deficiency; contacts with patients with aids, tuberculosis and other infectious diseases; infectious respiratory disease; sexually transmitted diseases; and parasitosis. The clients were followed up on average for a period of 3.2 months and received 2.4 visits, and $47.7 \%$ of them abandoned treatment. The students participated actively in the research, the methodology and the quantitative information obtained permitted the assessment of the quality of care, with the health service corresponding to the expectations for an institutional alternative for training and for providing care closer to the community.

UNITERMS: Outcome and Process Assessment (Health Care). Medical Care. Education, Medical. 


\section{REFERÊNCIAS BIBLIOGRÁFICAS}

1 - AMATO NETO V; MEDEIROS EAS; KALLÁS EG; LEVI GC; BALDY JL $S$ \& MEDEIROS RSS. AIDS na prática médica. Sarvier, São Paulo,1996.

2 - BRASIL. MINISTÉRIO DA SAÚDE, Secretaria de Projetos Especiais de Saúde e Coordenação Nacional de DST e AIDS. A epidemia de AIDS no Brasil: situação e tendências. Brasília, 1997.

3 - MATIDA LH. Aids de transmissão materno-infantil: análise de sobrevivência dos casos notificados ao sistema oficial do Estado de São Paulo de 1987 a 1994. Bol Epidemiol C.R.T. - DST/AIDS, C.V.E., Secretaria de Estado da Saúde, S.P., 16, (1): 5-10, mar 1998

4 - BRASIL. MINISTÉRIO DA SAÚDE. Tendência da epidemia. AIDS - Bol Epidemiol (2): 5, mar/maio 1998.

5 - ORTÚZAR MENESIA E. Estudo epidemiológico sobre a síndrome de imunodeficiência adquirida (AIDS) no município de Ribeirão Preto, S.P., Brasil. Dissertação de Mestrado, Departamento de Medicina Social, Faculdade de Medicina de Ribeirão Preto da USP, Ribeirão Preto, 1999.

6 - RUFFINO NETTOA; CARDOSO RAS; ALMEIDA VA\& FERREIRA IR. Co-positividade, Co-negatividade e precisão do teste de Elisa na detecção de anticorpos anti HIV-1. Medicina, Ribeirão Preto, 27: 353-55, 1994.

7 - BRASIL, MINISTÉRIO DA SAÚDE. Alternativas assistenciais à Aids no Brasil: as estratégias e resultados para a implantação da rede de Serviço de Assistência Especializada (SAE). AIDS - Bol Epidemiol (23-24): 7-13, jun/ago 1998.
8 - OLIVEIRA SN; HENNEMANN GV; FERREIRA FLF; AZEVEDO SA \& FORSTER AC. Avaliação epidemiológica da hanseníase e dos serviços responsáveis por seu atendimento em Ribeirão Preto - S.P., no ano de 1992. Medicina, Ribeirão Preto, 29: 114 - 122, 1996.

9 - SETUBAL S; TAVARES W \& OLIVEIRA SA. Influence of immunopreventable diseases and aids on the demand of an Infectious Diseases Department in Rio de Janeiro State, Brazil, in the course of thirty years (1965 - 1994). Rev Inst Med Trop São Paulo, 40: 185-192, 1998.

10 - CÉSAR LGC \& TANAKA OY. Inquérito domiciliar como instrumento de avaliação de serviços de saúde: um estudo de caso na região sudoeste da área metropolitana de São Paulo, 1989 - 1990. Cad Saúde Pública, Rio de Janeiro, 12, 59 - 70, 1996. Supl.2.

11 - GIR E; DUARTE G; MARTINEZ R; MORIYATM; FIGUEIREDO JF C; COSTA JC \& MACHADO AA. Expressão epidemiológica de outras doenças sexualmente transmissíveis entre portadores de AIDS. Rev Saúde Pública 28, 93 -99, 1994

12 - NUTBEAM D \& BLAKEY V. El concepto de promoción de la salud y la prevención del sida. Una base integrada y general para la acción en los años noventa. In: Promoción de la salud: una antologia. OPS, Washington, D.C. 1996. (Publicación Científica, 557).

Recebido para publicação em 05/05/99

Aprovado para publicação em 14/09/99 